\title{
Effect of Carvacrol on Inflammatory Factors and Myelin Repair in Experimental Autoimmune Encephalomyelitis on Female Lewis Rats
}

\author{
Mahdie Ahmadi \\ Islamic Azad University
}

Akram Eidi

Islamic Azad University

Mojtaba Khaksarian (D mojkhaksar@yahoo.com )

Lorestan University of Medical Sciences

Hassan Ahmadvand

Lorestan University of Medical Sciences

Fattah Sotoodehnejadnematalahi

Islamic Azad University

\section{Research Article}

Keywords: Remyelination, EAE, Multiple Scelrosis, Carvacrol, Inflammation

Posted Date: April 27th, 2021

DOI: https://doi.org/10.21203/rs.3.rs-430600/v1

License: (9) This work is licensed under a Creative Commons Attribution 4.0 International License. Read Full License 


\section{Abstract}

Carvacrol is a phenolic monoterpene constituent of essential oils produced by plants such as Nigella sativa. This study examined the therapeutic effects of carvacrol in a model of experimental autoimmune encephalomyelitis (EAE). EAE induction was performed on female Lewis rats and, after appearance of the first clinical signs, a second EAE group received carvacrol intraperitoneally each day for 17 days. Clinical symptoms and body weight were assessed daily. All animals sacrificed and histological and gene expression analysis of the spinal cord were performed. The carvacrol treated group scored lower for disease after therapeutic administration than the control group. Gene expression analysis on the carvacrol treated group showed a decrease in TNF-a, NF-ҚB and IL-17 (pro-inflammatory) gene expression and an increase in MBP and OLIG2 remyelination markers. In the carvacrol treated group, H\&E stain of spinal cord sections revealed a remarkable decrease in the amount of inflammatory cell infiltration. Immunostaining of lumbar spinal cord sections demonstrated a remarkable increase in MBP and a decrease in IL-17 secretion in the carvacrol treated group. These results demonstrate that carvacrol had a beneficial therapeutic effect and anti-inflammatory properties that significantly reduced the clinical scores and promoted myelin repair.

\section{Introduction}

Carvacrol is a monoterpenoid phenol that has special antioxidant effects against free radicals. Carvacrol has antioxidant, anti-inflammatory and antimicrobial properties. It has been shown to reduce the expression of EGF, TNF- $a$ and MCP-1 in patients exposed to mustard gas(Aristatile, Al-Assaf et al. 2013) In hepatotoxicity induced by D-galactosamine, carvacrol was found to reduce mRNA expression of IL-6, inducible nitric oxide synthase (iNOS), TNF-aand NF-ҚB(Aristatile, Al-Assaf et al. 2013). In IL-1ß-inflamed chondrocytes, carvacrol has been shown to inhibit the production of nitric oxide and prostaglandin E2 (PGE2) and reduce the iNOS and COX-2 expression. Inhibition of matrix metalloproteinase-13 (MMP-3) and MMP-3 genes and inhibition of NF-ҚB signaling pathway activation are other effects of carvacrol treatment(Yu, Zhang et al. 2012).

The neuroprotective effects of carvacrol in neurological disorders such as ischemia, epilepsy(Baluchnejadmojarad and Roghani 2016) and Parkinson's disease(Baluchnejadmojarad, Hassanshahi et al. 2014; Haddadi, Rajaei et al. 2018) have been demonstrated. In a Parkinson's model, carvacrol-treated mice showed improved memory deficits and decreased caspase(Dati, Ulrich et al. 2017) levels(Baluchnejadmojarad, Hassanshahi et al. 2014; Haddadi, Rajaei et al. 2018). In a cerebral ischemic model, carvacrol improved learning ability and memory in mice and decreased lipid peroxide and cell death biomarkers in the ischemic brain tissue. It also inhibited ferroptosis by increasing the expression of glutathione peroxidase 4 (GFX 4)(Guan, Li et al. 2019).

Multiple sclerosis (MS) is a neurodegenerative disease of the central nervous system (CNS), Which is known for features such as inflammation, altered axonal function and demyelination (Yoshida, Kimura et al. 2012). Experimental Autoimmune Encephalomyelitis (EAE) is a common model used to study MS. EAE 
is a complex condition in which the interaction of a variety of neuronal-damaging mechanisms and the immune system shows striking resemblance to key pathogenic features of MS. These features include inflammation, demyelination, axonal removal and gliosis. Regulatory mechanisms of inflammation and remyelination also occur in $\mathrm{EAE}$, so they act as a model for these processes.

EAE has a complex pharmacology and many drugs that are commonly used or are in development for MS have been tested and approved using an EAE model. However, a limited number of studies have evaluated the effect of carvacrol on MS patients or using the EAE model. An examination of the effect of carvacrol in an EAE model and by culturing spleen cells found that it can decrease clinical scores and IL6 , IFN- $\gamma$ and IL-17secretion and increase IL-10 and TGF- $\beta$. The carvacrol-treated mice also experienced less weight loss(Mahmoodi, Amiri et al. 2019).

This current study evaluated the inflammatory factors of IL-17, MCP, TNF-a, NF-ҚB and the genes involved in myelin repair (MBP, PDGFR and OLIG2) to determine the effects of carvacrol on the process of myelination and in reducing demyelination and inflammation by means of molecular and histological examinations of the spinal cords of Lewis rats.

\section{Materials And Methods}

\section{Induction, treatment of EAE and Clinical score}

Adult female Lewis rats weighting 180 to $210 \mathrm{~g}$ were used in this experiment. The animals were kept in clean conditions under a 12:12 $\mathrm{h}$ dark/light cycle and the room temperature was controled. The immunization protocol from Beeton et al(Beeton, Garcia et al. 2007) was used to prepare guinea pig spinal cord homogenate in distilled water, which then was mixed with complete Freund's adjuvant (CFA; Sigma-Aldrich; USA) containing Mycobacterium lyophilized cell powder.

At first, the rats were injected subcutaneously with $0.5 \mathrm{ml}$ of the mentioned emulsion at the tail. After $2 \mathrm{~h}$ and $48 \mathrm{~h}$, each rat received an intraperitoneal injection of pertusis toxin (Sigma Aldrich; USA) in distilled water.

After immunization, the rats were weighed daily and the clinical signs of EAE were monitored daily. The scores were as follows: 0 = without clinical signs; 1 = full tail paralysis; $2=$ moderate paralysis of hind limbs; 3 = hind limb paralysis; 4 = bilateral hind limb paralysis; 5 = complete palsy, dying or death. After monitoring for signs of disease on day 10 of post-immunization, the animals in the carvacrol treated group received $20 \mathrm{mg} / \mathrm{kg}$ carvacrol once daily for 17 days, from day 12 to day 28 post-immunization. The healthy control group received phosphate buffered saline (PBS). The EAE group of immunized rats received no treatment.

\section{Histological assessment}

After anesthetization, the rats were perfused intracardially with PBS and paraformaldehyde. Their lumbar spinal cords were removed and fixed overnight at $4^{\circ} \mathrm{C}$ in $4 \%$ paraformaldehyde. For inflammatory cell 
infiltration evaluation with H\&E stain, dehydration of fixed tissues was performed in an alcohol series, incubated in xylene until clear, embedded in paraffin and sectioned in $5-\mu \mathrm{m}$ thicknesses using a microtome. The paraffin-embedded sections were rehydrated in an alcohol series, stained with hematoxylin and then cleared in two baths of xylene. Then, the slides were washed and stained with eosin. These slides then were cover-slipped and photographed through a light microscope.

\section{Immunohistofluorescence}

As in previous histological studies, the lumbar spinal cords were isolated, fixed in paraformaldehyde, dehydrated in alcohol, incubated in xylene and embedded in paraffin. Sections of $6.8 \mu \mathrm{m}$ in size were prepared using a microtome. These were blocked with normal serum and stained with primary antibodies against myelin basic protein (MBP) and IL-17 (Abcam; UK; dilution 1:50). The slides were washed with PBS and incubated with goat anti-mouse rhodamine conjugate (Invitrogen; 1:100) and goat anti-rabbit FITC (1:100). The nuclei were stained with DAPI and images were obtained using a BX-51 fluorescent microscope (Olympus DP72).

\section{RNA extraction and quantitative RT- PCR}

The total RNA was isolated from the lumbar spinal cord with Trizol reagent (Parstous Company, Iran). The integrity of the isolated RNA was confirmed by agarose gel electrophoresis. To determine the purity and concentration of the RNA, a spectrophotometer (Pharmacia Biotech, Cambridge, United Kingdom) was used. The cDNA was synthesized using a cDNA synthesis kit (Ana Cell; Iran). RT- PCR was carried out using RT-PCR master mix (Amplicon). The thermal conditions were as follows: preheating for $3 \mathrm{~min}$ at $95^{\circ} \mathrm{C}$; denaturation for $30 \mathrm{~s}$ at $95^{\circ} \mathrm{C}$; annealing for $30 \mathrm{~s}$ at $60^{\circ} \mathrm{C}$; extension for $20 \mathrm{~s}$ at $72^{\circ} \mathrm{C}$.

The primers were designed and synthesized (Bioneer; Korea) to assessthe expression of GAPDH (housekeeping gene), Interleukin-10 (IL-10), IL-4, IL-1, IL-17, tumor necrosis factor a (TNFa), forkhead box p3 (FoxP3), tumor growth factor $\beta$ (TGF $\beta$ ), iNOS, nuclear factor-kappa B (NF-kB), platelet-derived growth factor receptor a (PDGFRa), Nrf2, MCP-1, MBP, nerve growth factor (NGF), brain-derived neurotropic factor (BDNF), OLIG2 and heme oxygenase 1( HO 1). The primer pairs used in this study are shown in table 1. For analysis of the relative gene expression level, $2^{-\Delta \Delta C t}$ formula was used.

\section{Statistical analysis}

The gene expression and histological data were analyzed by one-way ANOVA or t-student testing and compared using the Tukey post-test at $p<0.05$ as the significant difference.

\section{Results}

\section{Histopathological findings}

The carvacrol treated group received carvacrol from day 12 (peak disease score) to day28. The results indicate that daily administration of carvacrol during this period led to significant recovery (Fig. 1a) and 
significant recovery of all rats at day 24 . After treatment with carvacrol, relapse of EAE severity was diminished, compared to EAE group.

Up to day 9, no significant differences were observed between weight of groups. After day 9, significant weight loss was observed in both the EAE and carvacrol treated groups. Weight loss continued at a slower rate in the EAE group until day 28. In the carvacrol treated group, on days 21 to 23 , the weight began showing an upward trend.

\section{Histological analysis}

The efficacy of carvacrol on EAE- related CNS pathology was assessed. To evaluate inflammatory parameters, the lumbar spinal cord sections from animals in all groups were stained with hematoxylin and eosin. After staining, the samples were assessed for the amount of immune cell infiltration.

Figure 2(a) shows normal stained white matter of the lumbar spinal cords of the control group.

Figure 2(b) shows the EAE group and reveals glial and immune cell proliferation and motor neuron shrinkage. Figure 3(c) shows the carvacrol treated group with mild glial and immune cell proliferation in the white matter.

In the carvacrol treated group, the amount of infiltrated immune cells in the CNS decreased compared to the EAE group (Fig. 2). These results corroborate the anti-inflammatory effect of carvacrol on EAE. The data suggests suppression of the immune and inflammatory processes by carvacrol.

Immunohistofluorescence studies using anti-MBP and anti-IL-17 antibodies on spinal cord sections of the EAE and carvacrol treated groups revealed enhanced expression of IL-17 and decreased MBP expression in the EAE group. In the carvacrol treated group, IL-17 secretion decreased (Figs. 7 and 8). After treatment with carvacol and immunostaining with anti-MBP antibody, MBP immunoreactivity increased in the carvacrol treated group compared to the EAE group.

Figure 3 indicates that, in the EAE group, the increase in fluorescence intensity due to the increase in IL-17 secretion compared to the control group is obvious and significant $(p<0.05)$. There was no significant difference between fluorescence intensity in the control and carvacrol treated groups $(p>0.005)$. In the carvacrol treated group, the fluorescence intensity and, consequently, the secretion rate decreased remarkably compared to the EAE group $(p<0.000)$.

Figure 4 shows MBP immunohistofluorescence markers by group. In comparison with the EAE group, the fluorescence intensity increased remarkably in the carvacrol treated group. $(p<0.001)$. Figure 6 compares the MBP expression based on fluorescence intensity. The intensity of fluorescence in the EAE group decreased significantly compared to the control group $(p<0.0001)$. The fluorescence intensity of the carvacrol treated group and, consequently, the expression ratio relative to the EAE group increased significantly $(p<0.0001)$.

\section{Carvacrol diminished IL-17, IL-1 and NF-ҚுB}


The carvacrol effect on expression of the inflammatory gene was evaluated using RT- PCR and the results are shown in Fig. 6. EAE induction caused a significant increase in IL-17 compared to the control group ( $p$ $<0.0001)$, but this decreased in the carvacrol treated group to even below that of the control group $(p<$ $0.0001)$. The same pattern of change was observed for IL-1 and NF-KB $(p<0.0001)$. Carvacrol administration reversed the mRNA level of IL-1 and NF-ҚB to below that of the control group.

EAE increased the expression of MCP at the mRNA level compared to the control group. The mRNA level of MCP in the carvacrol treated group was even higher than for the EAE group $(p<0.0001)$. The expression of TNF- $a$ in the EAE group was higher than in the control group $(p<0.05)$, but did not change in the carvacrol treated group. The expression of TNF in the EAE and carvacrol treated group was similar.

\section{Effect of carvacrol on MBP, PDGFR and OLIG2}

The expression pattern of MBP, OLIG2 and PDGFR were assayed following EAE induction and in the carvacrol treated group. A decrease in MBP gene expression was observed in the EAE group $(p<0.05)$, but carvacrol significantly increased this level in the carvacrol treated group compared to the EAE and control groups $(p<0.0001)$.

The tissues were evaluated for expression of OLIG2 and it was found that the level of OLIG2 mRNA decreased in the EAE group and significantly increased in the carvacrol treated group compared to the EAE group. This level was even higher than for the control group.

Compared with the control group, PDGFR gene expression increased in the EAE group $(p<0.0001)$ and the PDGFR mRNA level decreased in the carvacrol treated group compared to the EAE group $(p<0.0001)$. No significant difference was observed between the carvacrol treated group and the control group $(p=$ 0.652; Fig. 7).

\section{Discussion}

Carvacrol has been shown to be effective on some inflammatory and neurodegenerative diseases of the brain, which makes it a good option for the treatment of MS. The characteristics of the EAE model were confirmed by the observed increase in neurological defects and clinical scoring, the presence of CNSinfiltrated immune cells and axonal degradation, which are consistent with results of previous studies(Mohajeri, Sadeghizadeh et al. 2015; Yang, Zheng et al. 2017).

Carvacrol has neuroprotective effects that are demonstrated in neurological models such as ischemia and Parkinson's disease(Baluchnejadmojarad, Hassanshahi et al. 2014; Dati, Ulrich et al. 2017). The carvacrol treatment results in the present study showed a decrease in clinical scores and histological changes. It also resulted in decreased expression of some proinflammatory genes and increased expression of genes involved in myelination.

CNS repair is the goal of treatment for CNS and autoimmune disorders. Different neuroprotective therapeutic strategies have been used in clinical trials but, as yet, no comprehensive and successful 
examination of remyelination has been proposed. In the case of MS, remyelination not only better protects from axonal damage, but also can halt progression of the disease.

Oligodendrocytes have been a focus of attention for the development of therapeutic approaches and treatments for neurodegenerative disease because of their myelination potential(Kotter, Stadelmann et al. 2011). One of the most important features of MS is demyelination, in which the axon-oligodendrocyte relationship in the CNS is disrupted(Shakhbazau, Schenk et al. 2016). Evaluation of the therapeutic effects of such research methods requires the identification of oligodendrocytes. For this purpose, the present study used MBP, PDGFR and OLIG2 markers.

The results of molecular analysis for the carvacrol treated group showed that the remyelination rate increased and demonstrated that carvacrol increased OLIG2 mRNA expression. It was found that clinical scoring in the carvacrol treated group was lower than in the EAE group, indicating that the clinical damage improved with carvacrol treatment. OLIG2 expression increased in the carvacrol treated group as the clinical scores decreased. OLIG2 encodes the transcription factor that is necessary for remyelination and is obtained through proliferation and differentiation of neuronal progenitors and oligodendrogenesis progenitor cells (OPCs). This factor is necessary for oligodendrogenesis in the spinal cord. OLIG2expressing progenitor cells differentiate into oligodendrocytes in damaged lesions, leading to premature myelination of the CNS(Wegener, Deboux et al. 2015) .

In addition to OLIG2, PDGF is also involved in activating the oligodendrogenesis pathway and is involved in their proliferation. An increase in PDGF has been shown to increase the number of OPCs in demyelinated lesions. However, although PDGF infusion induces SVZ type B cell proliferation which is effective for remyelination, it can have side effects such as tumor formation(Rivera and Aigner 2012).

OPCs express various markers, the most important of which are NG2 and PDGFRa. Because NG2 can be present in other glia precursor cells, the PDGFRa marker is more specific for immature oligodendrocytes.

In this study PDGFRa was used to assess the amount of immature oligodendrocyte formation. Precursor oligodendrocytes were observed to attach to neurons and, after two to three days, myelinated them(Blakemore and Irvine 2008). However, contrary to the results of previous studies, PDGFR showed increased expression in the EAE group and decreased expression in the carvacrol treated group.

In agreement with the results of the present study, Qiang Zho et al. reported that transgenic mice showed that deletion of PDGFR caused precocious differentiation of OPCs. Their findings demonstrate that PDGFR is an important negative regulator of OPC maturation in the developing CNS. During OPC differentiation, PDGFR expression gradually decreases and is completely silent in adult oligodendrocytes. It is not clear how cessation of PDGFR expression or OPC signaling triggers oligodendrocyte differentiation. One possibility is that PDGFR signaling may inhibit the expression or function of oligodendrocyte maturation activators. These activators include myelin regulatory factor (MRF), sip1 and SOX10, each of which is sufficient to differentiate oligodendrocytes. If this hypothesis is correct, $N k \times 2.2$ transcriptional inhibitor initiates internal differentiation by removing the inhibition of these regulatory 
factors and responding to axon signals or other environmental messages. It may initiate internal differentiation by removing the inhibition of these regulatory factors in response to axon signals.

$N k x 2.2$ is a transcriptional inhibitor in cell-fate control during CNS development and determines the time of OPC differentiation. Overexpression of $N k x 2.2$ in transgenics will extinguish PDGFR expression. It is possible that, when $N k x 2.2$ binds to the binding site on the PDGFR promoter, it inhibits PDGFR transcription. By repressing the expression of the membrane receptor PDGFR, the OPCs will no longer respond to mitogen PDGFR, which will cause the proliferation and activation of cell differentiation to stop.

On the other hand, an increase in Nkx2.2 expression and inhibition of PDGFR expression has been observed to increase MBP expression in primary OPCs. Therefore, the possibility that Nkx2.2 may influence other factors simultaneously to affect differentiation and maturation of OPCs cannot be ignored (Zhu, Zhao et al. 2014). After demyelination, OPCs switch from neutral to active and mitotic cells by upregulating OLIG2 and Nkx2.2 (Göttle and Küry 2015). In general, the reduction in PDGFR expression by carvacrol in this study could provide a pathway for the differentiation of OPCs and help to myelinate and repair damage caused by the model induction.

MBP plays the most important role in the formation of the three-dimensional (3D) structure of the axial membrane of myelin. This protein binds to membrane lipids as it enters the oligodendrocyte membrane and strengthens the 3D structure of the myelin membrane(Czepiel, Boddeke et al. 2015). MBP was used in the present study as an adult oligodendrocyte marker to evaluate the final remyelination rate. The goal was to determine which changes occur during the process of remyelination from immature oligodendrocytes to adults. MBP mRNAs are located at one point in the cytoplasm of oligodendrocytes before myelination and then disperse into the cytoplasm at the beginning of myelination. This spatial separation differentiates oligodendrocytes into complete myelinating cells (Musse and Harauz 2007) .

It has been shown in MS patients and animal models that MBP decreased about three-fold compared to normal levels. In glial cells and macrophages, the group of enzymes called protein arginine deiminase (PAD) becomes uncontrolled for unknown reasons in inflammatory diseases such as MS and produces immunogenic citrullinated epitopes. PAD2 and PAD4 levels increase in MS. It is possible that these two enzymes alter the structure of the third MBP, and predispose them to proteolysis so that MBP cannot properly form the multilayer structure of a myelin sheath. Eventually, the accumulation of nerve cells will decrease(Jones, Causey et al. 2009).

In our study induction of the EAE model reduced MBP expression in the EAE group and further study showed that carvacrol administration increased MBP expression in the carvacrol treated group. These results were confirmed by both molecular methods and immunohistofluorescence analysis. The increase in OLIG2 expression increased the production of differentiated oligodendrocytes as well as MBP expression, which indicates the activity of oligodendrocytes in the myelination pathway. The increase of these two factors demonstrates the process of myelination. However, molecular processes are so 
complex that these observations cannot easily be attributed to an increase or decrease in the expression of a gene. In such cases, many factors must be considered.

Studies have shown that carvacrol has an effect on inflammatory factors and oxidative stress. Gholijani et al. (2016) caused inflammation in mice and cultured their spleen cells to show that carvacrol reduced the formation of IL-17 as well as the expression of T-bet, GATA-3 and ROR $\llbracket c$ transcription factors that necessary for the maturation and function of Th1, Th2 and Th17 cells, respectively)(Gholijani and Amirghofran 2016). The Th17 lymphocyte has multiple pathogenic roles, most of which are attributed to IL-17 secretion. These include neutrophil recall, activation of innate immune cells, increased B lymphocyte function and stimulation of the release of inflammatory cytokines, including IL-1 $\beta$ and TNF- $\alpha$, which are the cause of many autoimmune diseases and the induction of EAE and are present in many MS lesions. IL-1 acts directly on T lymphocytes and causes the secretion of IL-17; therefore, it plays a pivotal role in the progress of autoimmunity in EAE and initiates the differentiation of pathogenic Th17 cells(Sutton, Brereton et al. 2006). Th17 causes BBB disruption and promotes CNS immune cell traffic and tissue inflammation through IL-17 and IL-22. II-17 exerts its pathological effects through chemokine stimulation and the adhesin molecules involved in the neutrophils penetration from the surrounding environment to the CNS(Raphael, Nalawade et al. 2015) .

Due to the decrease in IL-17 expression caused by carvacrol both in RT and immunohistochemical histological analysis in the present study, it appears that the effectiveness of carvacrol on reducing IL-17 production may relate, at least partially, to the modulation of auto-reactive Th17 and Th1. Carvacrol also diminish the flow of leukocytes to the CNS by reducing the inflammatory cytokines that are participated in the production of chemokines and adhesion molecules(Yang, Zheng et al. 2017). Studies have shown that carvacrol reduces IL-1 and TNF- $\alpha$ and inhibits dendritic cell maturation and function as well as the selection of T cell responses.

The results of this research on the NF-ҚВ and IL-1 gene expression are consistent with the results of Gholijani et al. and indicated that carvacrol reduced the expression of these genes(Raphael, Nalawade et al. 2015). NF-ҚB plays an important role in inflammatory processes. Aristatile et al. Induced inflammation in rat liver cells and showed that treatment of these cells with carvacrol reduced NF-K $\beta$ and TNF- $\alpha$ expression(Aristatile, Al-Assaf et al. 2013). The TNF- $\alpha$ gene promoter contains the NF-K $\beta$ response element. Under normal condition, NF- $\beta$ is present in the cytoplasm of cells and binds to IҚBa and ІҚВB, which prevents it from entering the nucleus. Separation of NF-ҚB from ІҚB leads to NF-ҚB entry into the nucleus and its binding to specific sequences in the promoters of specific genes and triggers gene expression. Aristatile et al. showed that carvacrol inhibits NF-ҚВ expression by inhibiting ІҚВ degradation, which inhibits TNF-a gene expression(Gimenez, Sim et al. 2006).

When EAE mice are immunized with myelin peptides, increased TNF-a expression exacerbates demyelination ${ }^{[27]}$. However, the results of analysis of TNF-a gene in the present study was not consistent with the results of previous studies. In the carvacrol treated group, the carvacrol did not change the expression of this gene compared to the EAE group. 
Despite the proinflammatory effect of TNF-a, which has been the subject of numerous articles, Arnet et al.,(Arnett, Mason et al. 2001) got unexpected results. They induced a demyelinated model in mice using cuprizone and deliberately inhibited TNF-a using XPro1595. Contrary to expectations, they found that the lack of TNFa caused significant delays in remyelination. These results were confirmed by histological and immunohistochemical analysis for myelin proteins. The reason for this failure in repair was shown to be the reduction of the pool of proliferating oligodendrocyte precursors, which resulted in a decrease in the number of adult oligodendrocytes.

TNF- $\alpha$ actually initiates remyelination through the TNFR2 signaling pathway and TNFR2 is necessary for the maturation of oligodendrocytes(Kircik and Del Rosso 2009). MCP inflammatory factor also is involved in the development of MS. It is broadly expressed in brain of MS patients, but not in the white matter of CNS tissue of healthy individuals. MCP is a $\beta$-chemokine that is a potent monocyte and T-cell chemoattractant and is secreted by active hypertrophic astrocytes(Van Der Voorn, Tekstra et al. 1999). Altered astrocyte functions, such as loss of normal activity or gaining abnormal functions could be involved in the onset and progression of MS(Mostafavi, Khaksarian et al. 2014). MCP activates and invokes myelin-destroying macrophages and is directly involved in the progression of MS.

MCP causes the secretion of lysosomal enzymes in monocytes and provides the signal needed to activate $T$ lymphocytes, thereby facilitating antigen presentation. On the other hand, it also increases the secretion of MMP-9 by T-cells. MMP-9 has the ability to destroy the basal membrane and other matrix compounds, which will cause inflammatory cells to migrate to such tissue. MCP- 1 can activate microglia cells in the tissue and in the newly arrived lymphocytes in MS wounds, causing tissue damage and demyelination. MCP-1 also increases inflammation, stimulates the secretion of enzymes, which is likely involved in BBB degradation and activates monocytes(Mostafavi, Khaksarian et al. 2014).

The effect of carvacrol on MCP as related to inflammatory factors in patients exposed to mustard gas has been shown to decrease. The antioxidant effect of carvacrol on MCP-1 also has been demonstrated in mice infected with Campylobacter jejuni. However, the results of this study did not agree with those results. On the contrary, carvacrol not only failed to reduce this factor in the carvacrol treated group, but actually increased it compared to the EAE group. Little study has been done thus far on the efficacy of carvacrol on MCP-1 in an EAE model and this finding requires further investigation. The favorable effects of carvacrol observed on some inflammatory factors and myelin repair factors show promise for the therapeutic effects of carvacrol. In this regard, additional clinical and molecular evidence must be gathered.

\section{Declarations}

\section{Funding}

This study is sponsored by Science and Research Branch, Islamic Azad University, Tehran, Iran with a grant no. of 488 
Declaration of Conflicting Interests

The author declares no conflict of interest, financial or otherwise.

Ethics approval and consent to participate

All stages of this experiment were in accordance with the ethical standards of the Ethics Committee of Lorestan University of Medical Sciences, Khorammabad, Iran (Approval ID: IR.LUMS.REC.1397.201).

Acknowledement

The authors gratefully thank Department of Biology, School of Basic Science, Science and Research Branch, Islamic Azad University, Tehran, Iran.

\section{References}

Aristatile B, Al-Assaf AH, et al. (2013). "Carvacrol suppresses the expression of inflammatory marker genes in D-galactosamine-hepatotoxic rats." Asian Pacific Journal of Tropical Medicine 6(3): 205-211

Arnett, H. A., J. Mason, et al. (2001). "TNFa promotes proliferation of oligodendrocyte progenitors and remyelination." Nature neuroscience 4(11): 1116-1122.

Baluchnejadmojarad, T., J. Hassanshahi, et al. (2014). "Protective effect of carvacrol in 6hydroxydopamine hemi-parkinsonian rat model." Journal of Basic and Clinical Pathophysiology 2(2): 2934.

Baluchnejadmojarad T, Roghani M (2016). "The protective effect of carvacrol on kainic acid-induced model of temporal lobe epilepsy in male rat." Journal of Basic and Clinical Pathophysiology 4(2): 11-16

Beeton C, Garcia A, et al. (2007). "Induction and clinical scoring of chronic-relapsing experimental autoimmune encephalomyelitis." JoVE (Journal of Visualized Experiments)(5): e224

Blakemore W and K. Irvine (2008). "Endogenous or exogenous oligodendrocytes for remyelination." Journal of the neurological sciences 265(1-2): 43-46

Czepiel, M., E. Boddeke, et al. (2015). "Human oligodendrocytes in remyelination research." Glia 63(4): 513-530.

Dati, L., H. Ulrich, et al. (2017). "Carvacrol promotes neuroprotection in the mouse hemiparkinsonian model." Neuroscience 356: 176-181.

Gholijani N, Amirghofran Z (2016). "Effects of thymol and carvacrol on T-helper cell subset cytokines and their main transcription factors in ovalbumin-immunized mice." Journal of immunotoxicology 13(5):

729-737 
Gimenez MA, Sim J, et al. (2006). "A tumor necrosis factor receptor 1-dependent conversation between central nervous system-specific $T$ cells and the central nervous system is required for inflammatory infiltration of the spinal cord." The American journal of pathology 168(4): 1200-1209

Göttle P and P. Küry (2015). "Intracellular protein shuttling: a mechanism relevant for myelin repair in multiple sclerosis?" International journal of molecular sciences 16(7): 15057-15085

Guan X, Li X, et al. (2019). "The neuroprotective effects of carvacrol on ischemia/reperfusion-induced hippocampal neuronal impairment by ferroptosis mitigation." Life sciences 235: 116795

Haddadi H, Rajaei Z, et al. (2018). "Chronic treatment with carvacrol improves passive avoidance memory in a rat model of Parkinson's disease." Arquivos de neuro-psiquiatria 76(2): 71-77

Jones J, Causey C, et al. (2009). "Protein arginine deiminase 4 (PAD4): Current understanding and future therapeutic potential." Current opinion in drug discovery \& development 12(5): 616

Kircik, L. H. and J. Q. Del Rosso (2009). "Anti-TNF agents for the treatment of psoriasis." Journal of drugs in dermatology: JDD 8(6): 546-559.

Kotter MR, Stadelmann C, et al. (2011). "Enhancing remyelination in disease-can we wrap it up?" Brain 134(7): 1882-1900

Mahmoodi, M., H. Amiri, et al. (2019). "Carvacrol ameliorates experimental autoimmune encephalomyelitis through modulating pro-and anti-inflammatory cytokines." Life sciences 219: 257263.

Mohajeri, M., M. Sadeghizadeh, et al. (2015). "Polymerized nano-curcumin attenuates neurological symptoms in EAE model of multiple sclerosis through down regulation of inflammatory and oxidative processes and enhancing neuroprotection and myelin repair." Neuropharmacology 99: 156-167.

Mostafavi H, Khaksarian M, et al. (2014). "Fluoxetin upregulates connexin 43 expression in astrocyte." Basic and clinical neuroscience 5(1): 74

Musse, A. A. and G. Harauz (2007). "Molecular "negativity" may underlie multiple sclerosis: role of the myelin basic protein family in the pathogenesis of MS." International review of neurobiology 79: 149172.

Raphael I, Nalawade S, et al. (2015). "T cell subsets and their signature cytokines in autoimmune and inflammatory diseases." Cytokine 74(1): 5-17

Rivera, F. J. and L. Aigner (2012). "Adult mesenchymal stem cell therapy for myelin repair in multiple sclerosis." Biological research 45(3): 257-268. 
Shakhbazau A, Schenk GJ, et al. (2016). "Demyelination induces transport of ribosome-containing vesicles from glia to axons: evidence from animal models and MS patient brains." Molecular biology reports 43(6): 495-507

Sutton C, Brereton C, et al. (2006). "A crucial role for interleukin (IL)-1 in the induction of IL-17-producing T cells that mediate autoimmune encephalomyelitis." The Journal of experimental medicine 203(7): 1685-1691

Van Der Voorn P, Tekstra J, et al. (1999). "Expression of MCP-1 by reactive astrocytes in demyelinating multiple sclerosis lesions." The American journal of pathology 154(1): 45-51

Wegener, A., C. Deboux, et al. (2015). "Gain of Olig2 function in oligodendrocyte progenitors promotes remyelination." Brain 138(1): 120-135.

Yang T, Zheng Q, et al. (2017). "Effect of catalpol on remyelination through experimental autoimmune encephalomyelitis acting to promote Olig1 and Olig2 expressions in mice." BMC complementary and alternative medicine 17(1): 1-15

Yoshida, H., A. Kimura, et al. (2012). "Low dose CP-690,550 (tofacitinib), a pan-JAK inhibitor, accelerates the onset of experimental autoimmune encephalomyelitis by potentiating Th17 differentiation." Biochemical and biophysical research communications 418(2): 234-240.

Yu H, Zhang Z-L, et al. (2012). "Carvacrol, a food-additive, provides neuroprotection on focal cerebral ischemia/reperfusion injury in mice." PloS one 7(3): e33584

Zhu Q, Zhao X, et al. (2014). "Genetic evidence that Nkx2. 2 and Pdgfra are major determinants of the timing of oligodendrocyte differentiation in the developing CNS." Development 141(3): 548-555

\section{Tables}

Table-1.List of RT-PCR primers 


\begin{tabular}{|c|c|c|}
\hline Gene symbol & Sequence $\left(5^{\prime} \rightarrow 3^{\prime}\right)$ & Accession ID \\
\hline \multirow[t]{2}{*}{ NF-K $\beta$} & F:CTATGACAGCAAAGCCCCCA & \multirow[t]{2}{*}{ NM_001276711.1 } \\
\hline & R:ACATCCGTGGGGGAAAAGTC & \\
\hline \multirow[t]{2}{*}{$\mathrm{IL}-17$} & F:CCATCCATGTGCCTGATGCT & \multirow[t]{2}{*}{ NM_001106897 } \\
\hline & R:AGGGTGAAGTGGATTGACGG & \\
\hline \multirow[t]{2}{*}{ Pdgfr- $\alpha$} & F:CAGACATTGACCCTGTTCCAGAGG & \multirow[t]{2}{*}{ NM_001143805 } \\
\hline & R:GAATCTATGCCAATATCATCCATC & \\
\hline \multirow[t]{2}{*}{$\mathrm{IL}-1$} & F:GGACCCAAGCACCTTCTTTT & \multirow[t]{2}{*}{ NM_031512.2 } \\
\hline & R:AGACAGCACGAGGCATTTT & \\
\hline \multirow[t]{2}{*}{ MCP-1 } & F:GGGCCTGTTGTTCACAGTTGC & \multirow[t]{2}{*}{ NM_031530.1 } \\
\hline & R:GGGACACCTGCTGCTGGTGAT & \\
\hline \multirow[t]{2}{*}{ TNF- $\alpha$} & F:CCGATTTGCCATTTCATACC & \multirow[t]{2}{*}{ NM_012675.3 } \\
\hline & R:GAGTCCGGGCAGGTCTACTT & \\
\hline \multirow[t]{2}{*}{ Olig2 } & F:TCAAGTCATCTTCCTCCAGCACCT & \multirow[t]{2}{*}{ NM_01100557.1 } \\
\hline & R:GGCTCAGTCATCTGCTTCTTGTCT & \\
\hline MBP & F:CTATAAATCGGCTCACAAGG & NM_001020462.1 \\
\hline
\end{tabular}

\section{Figures}




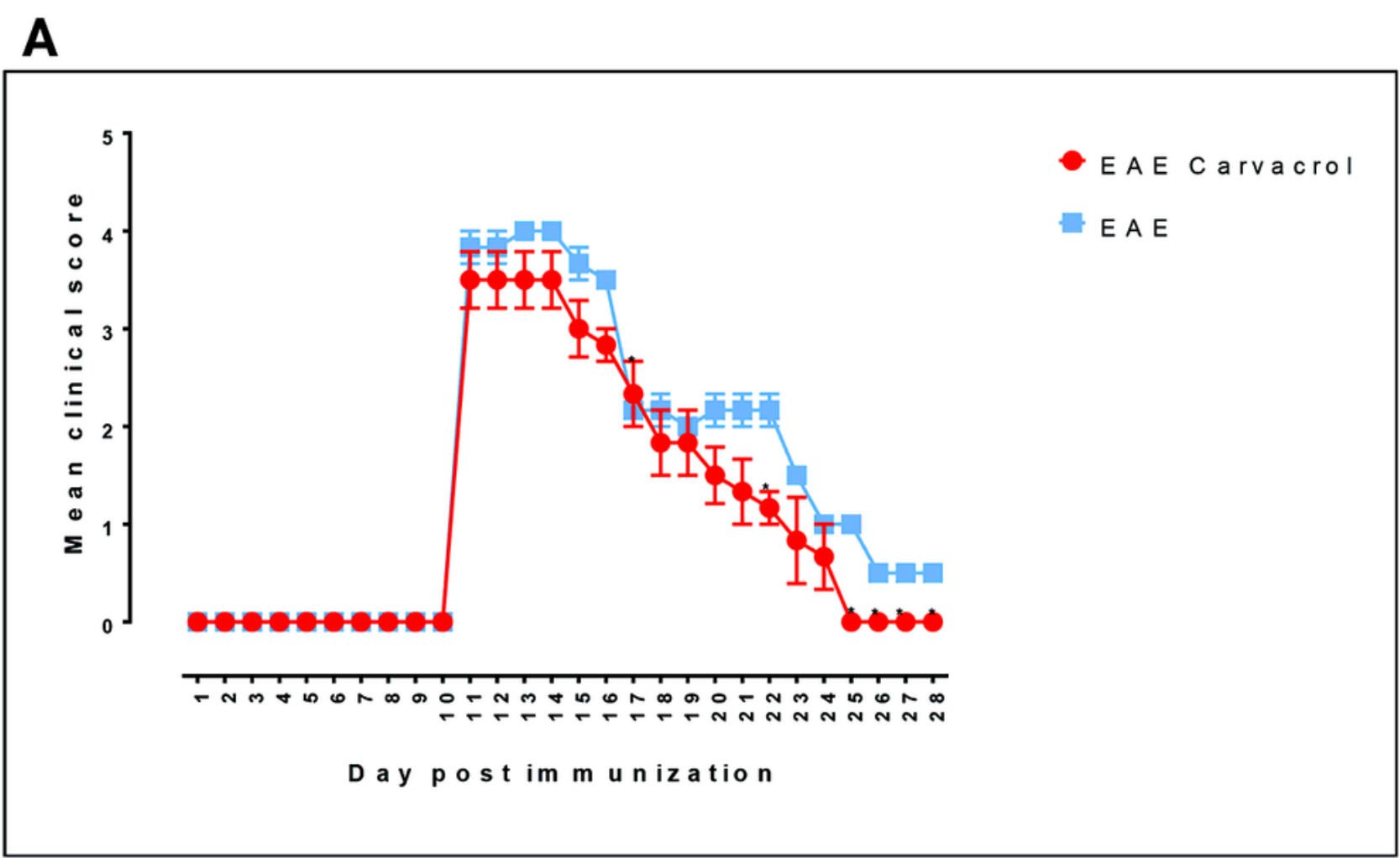

B

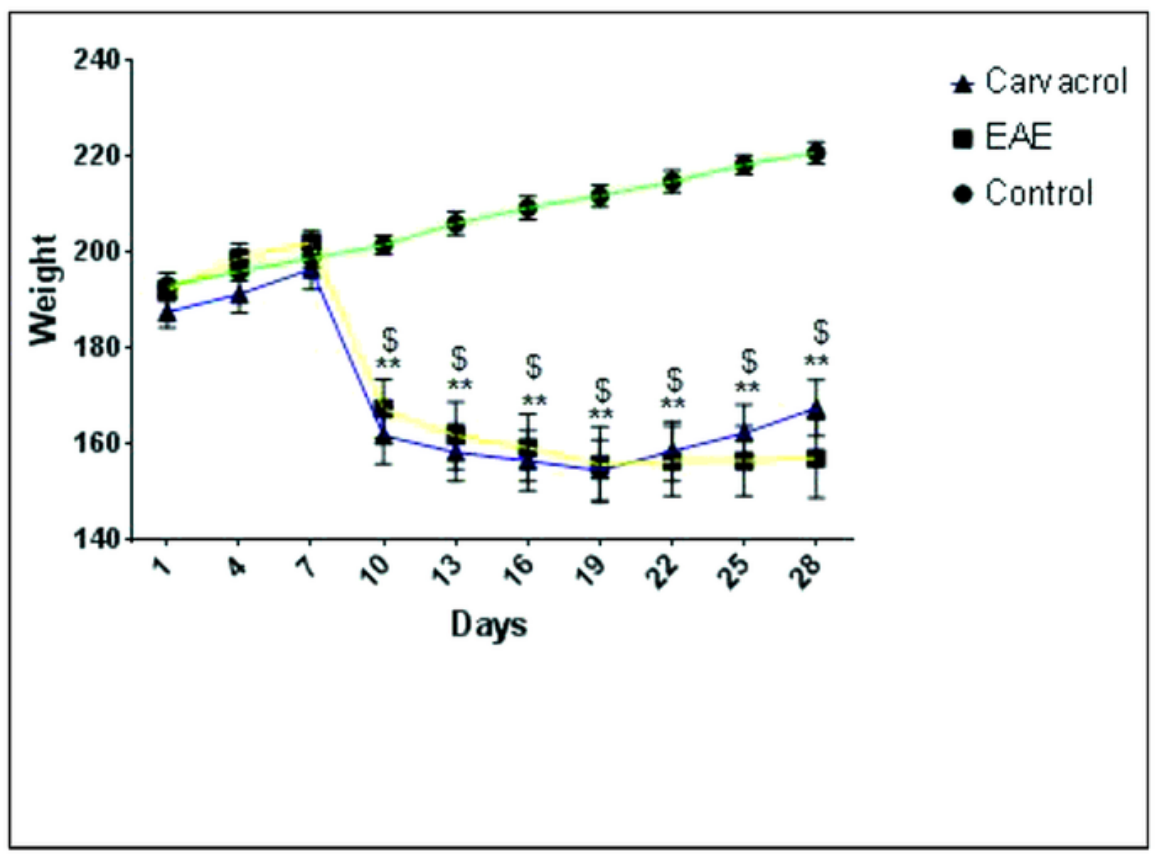

Figure 1

A) Mean daily scores of EAE severity in rats in each treatment group. Results are reported as the mean \pm SEM. ${ }^{*} p<0.05$ Significant difference with EAE group. B) Weight of rats $(\mathrm{g})$ in carvacrol treated group from day 0 to day 28. *, \$ Significant difference between carvacrol treated group and EAE group respectively with control group 

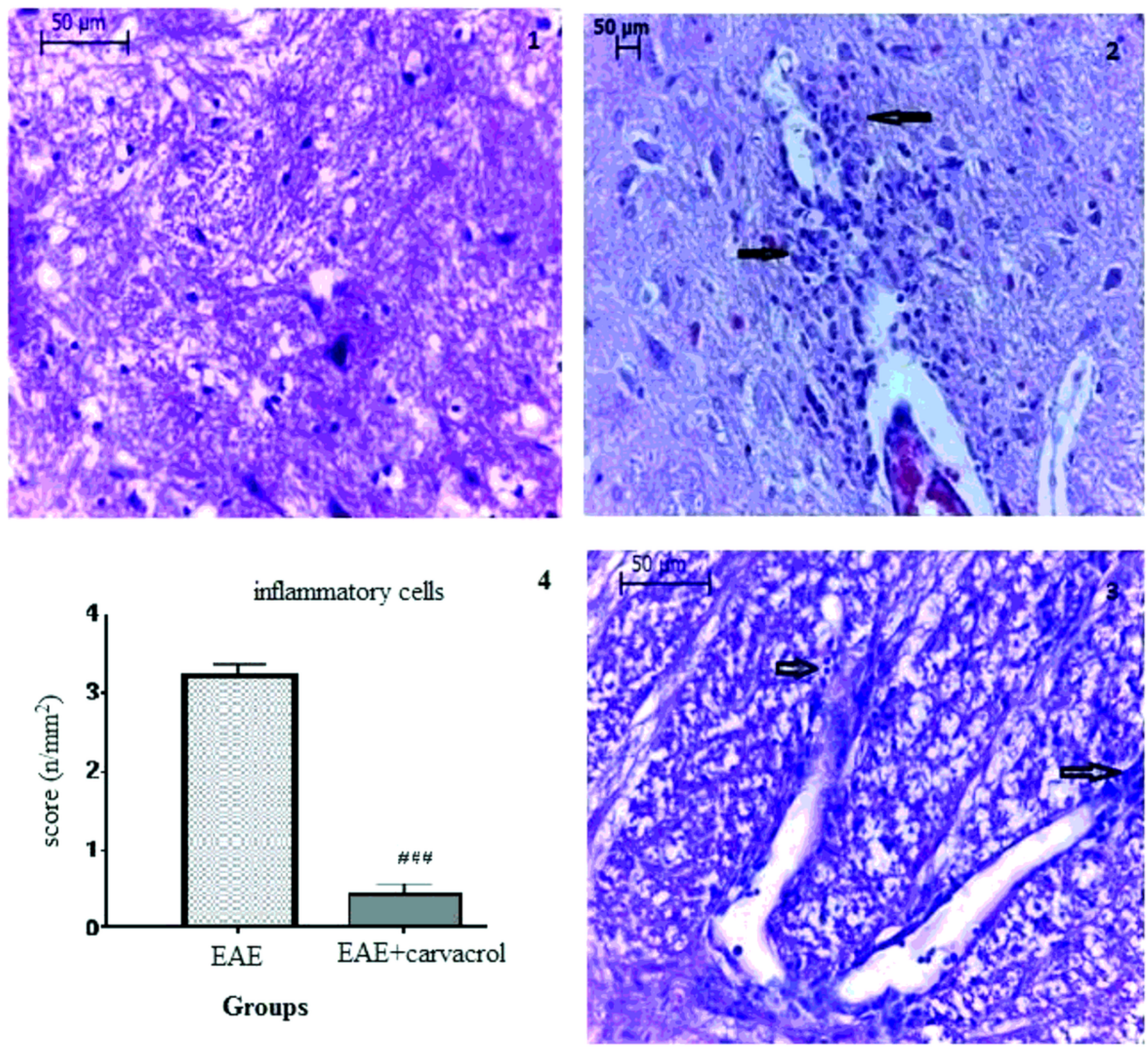

\section{Figure 2}

Infiltration of immune cells in lumbar spinal cord of animals in all groups. The arrows indicate the status of immune cells:. (1) control group; (2) EAE group; (3) carvacrol treated group group. (4) This image shows inflammatory cell influx into the CNS after carvacrol treatment. The amount of infiltration in the carvacrol treated group group was significantly lower than in the EAE group $(p<0.05)$. \#Significant differences with the EAE group $(P<0.05)$ 

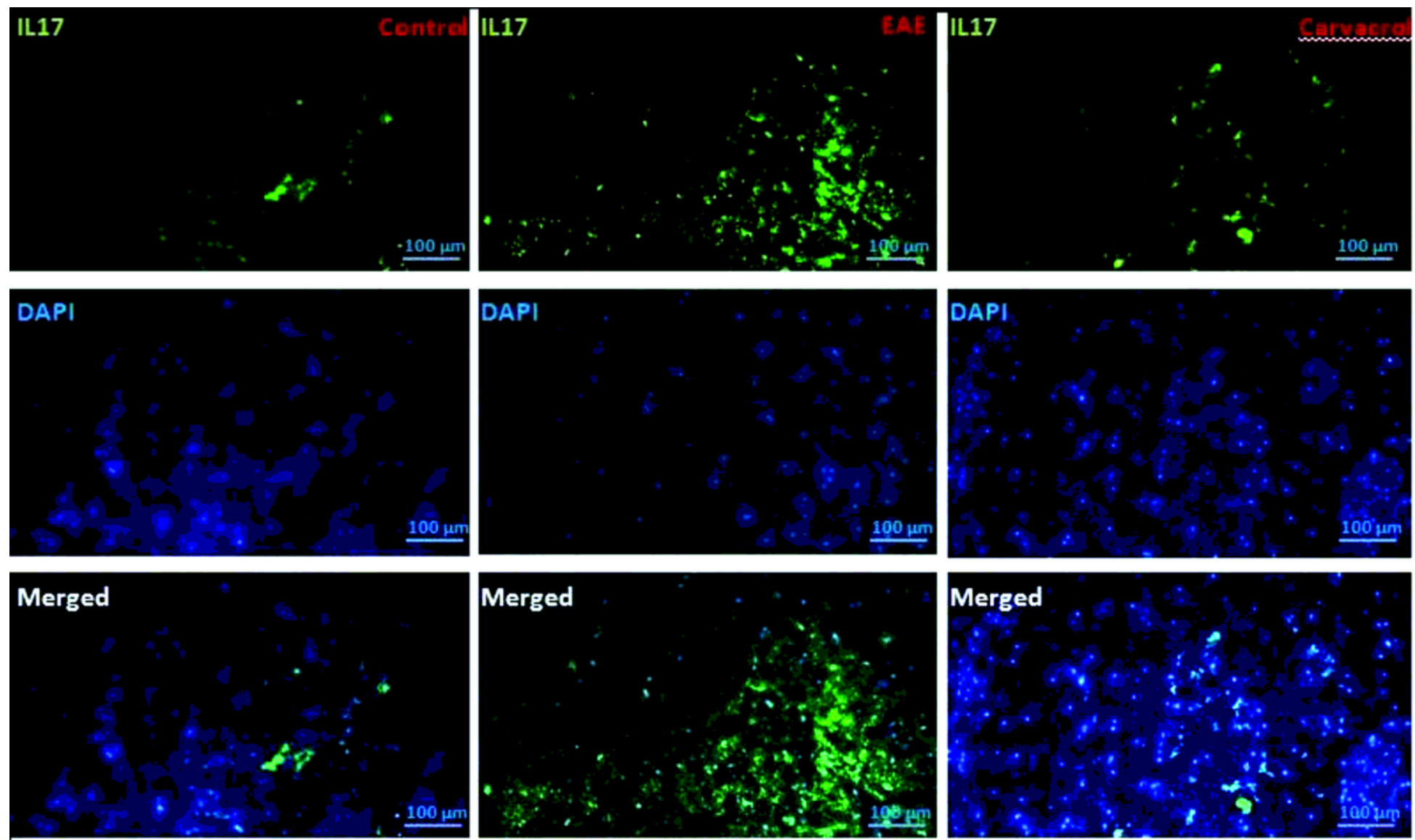

\section{Figure 3}

Immunohistofluorescence assay for IL-17 marker in: (a) control group; (b) EAE group; (c) carvacrol treated group. scale bar: $100 \mu \mathrm{m}$ 

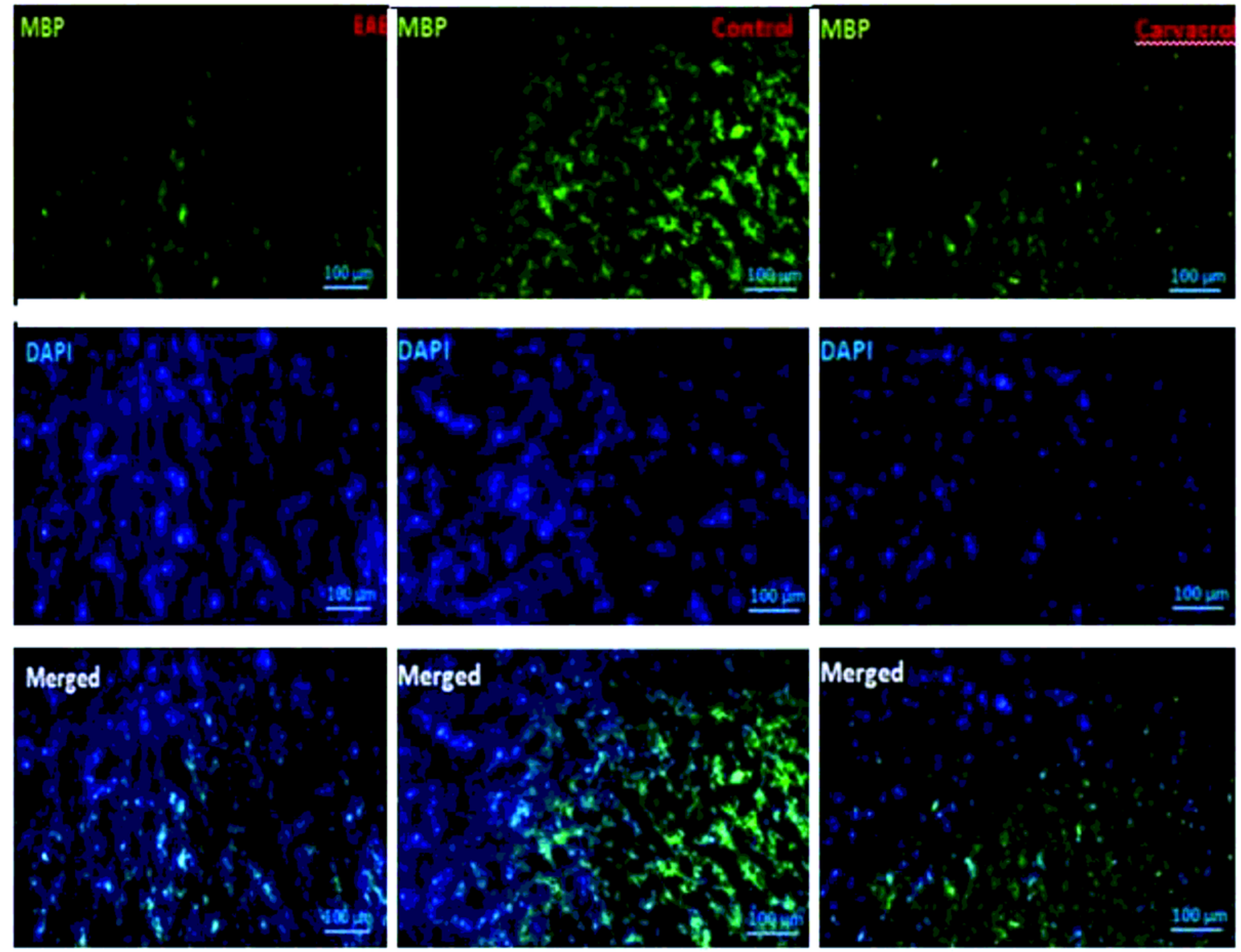

Figure 4

MBP immunohistofluorescence markers by group: (a) EAE; (b) control; (c) carvacrol treated group. scale bar: $100 \mu \mathrm{m}$ 


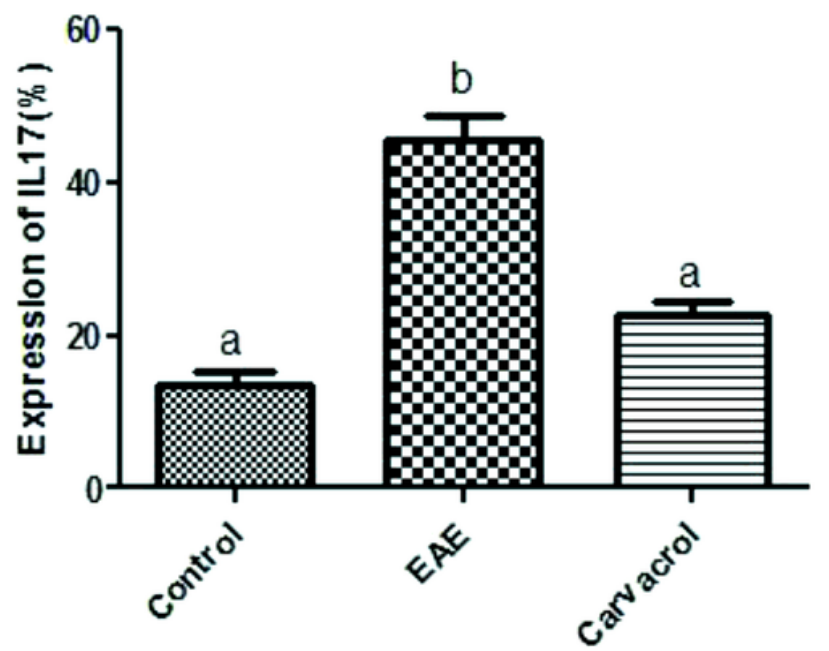

A

Groups

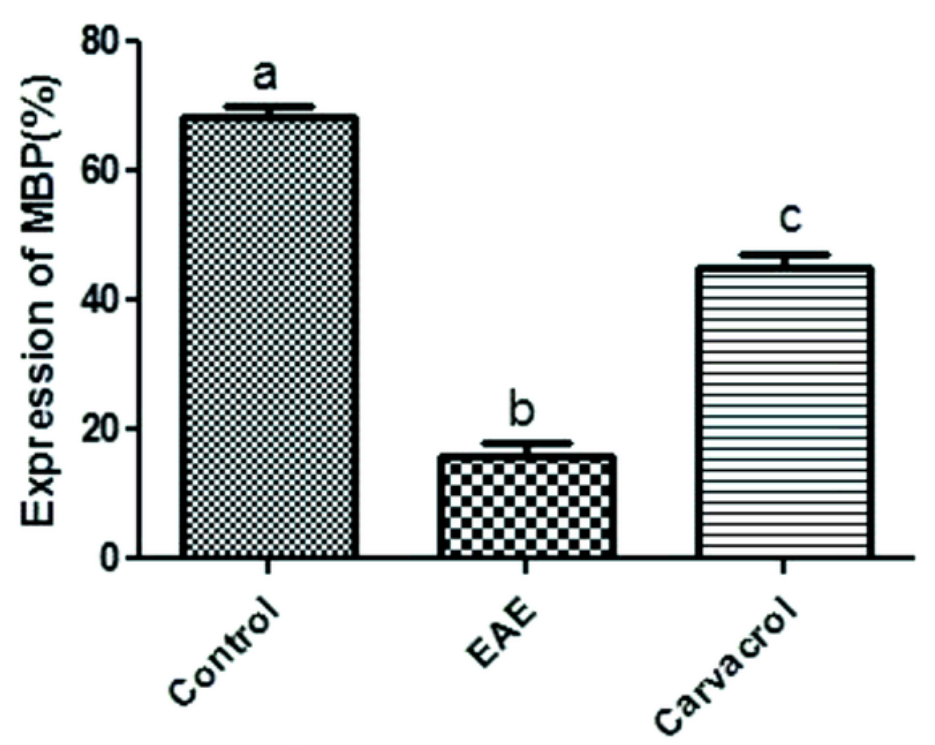

B Groups

Figure 5

A) Comparison of IL-17 expression based on fluorescence intensity. The intensity of fluorescent in the EAE group compared to the control group had a significant increase $(P<0.0001)$. In the carvacrol treatment group, the fluorescence intensity and consequently the secretion rate was significantly reduced compared to the EAE group $(\mathrm{P}<0.000)$. B) Comparison of MBP expression based on fluorescence intensity. Figures shows that the fluorescence intensity decreased significantly in the EAE group compared to the control group $(p<0.0001)$. In the carvacrol treated group, the fluorescence intensity and, consequently, the MBP expression ratio relative to the EAE group increased significantly $(p<0.0001)$. 


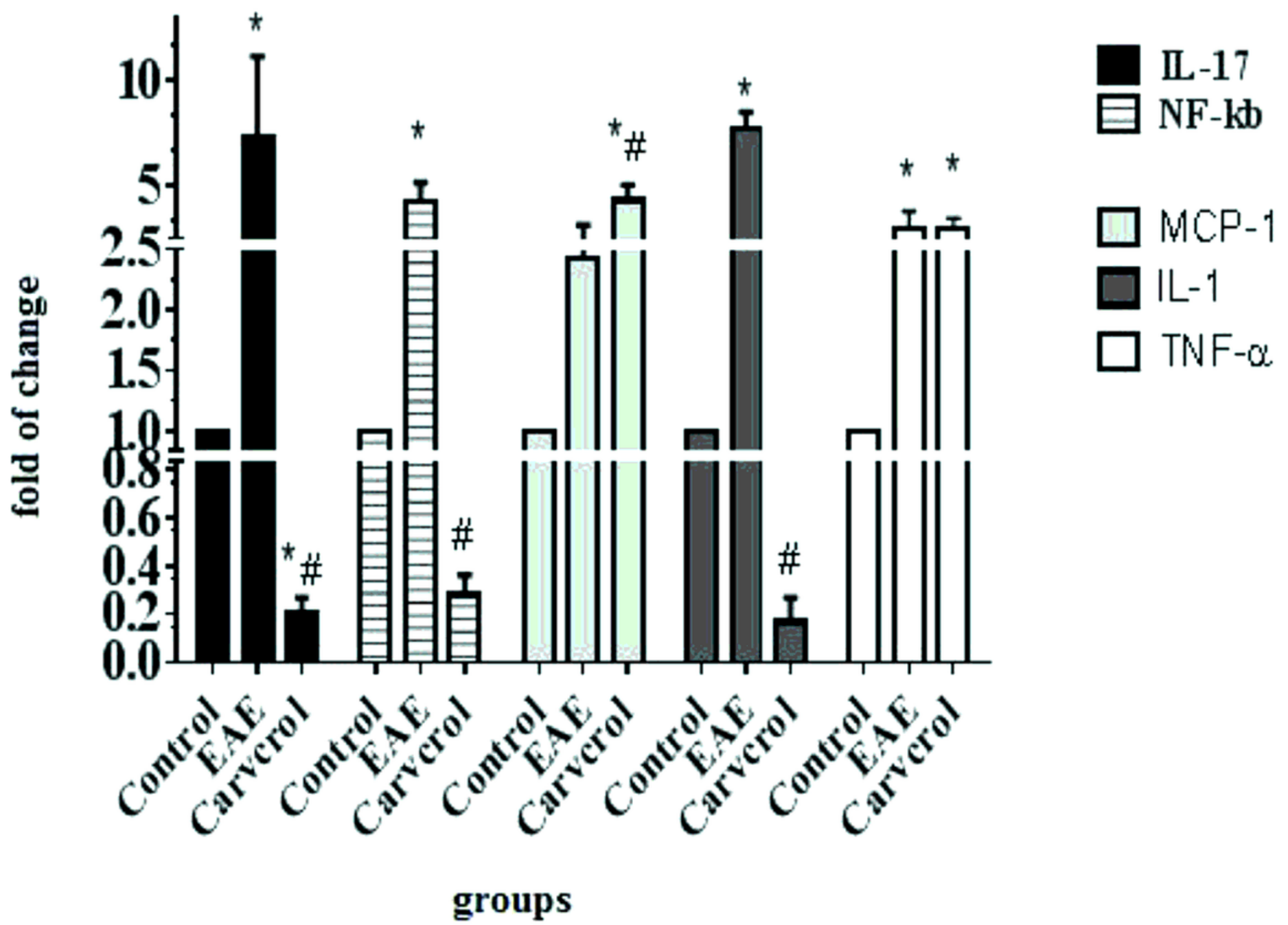

Figure 6

Effect of carvacrol on the gene expression of IL-17, NF-ҚВ, MCP-1 and IL-1 in the EAE group and carvacrol treated group compared to the control group. ${ }^{*}$, \#: Shows a significant difference compared to the control group and EAE group respectively. 


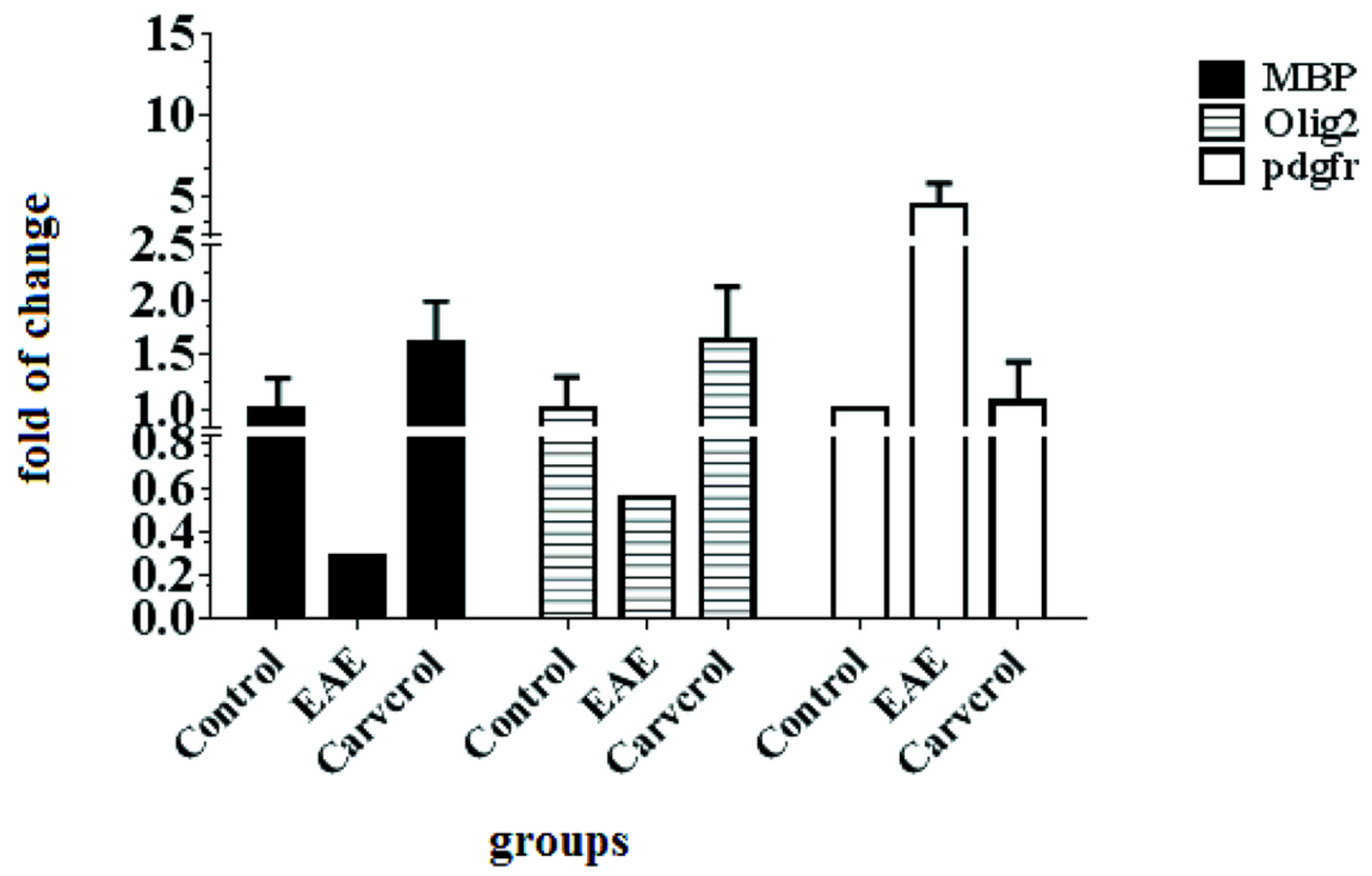

Figure 7

Effect of carvacrol on gene expression of MBP, OLIG2, PDGFR in EAE group and carvacrol treated group compared to control group. * and\#: Shows a significant difference compared to the control group and EAE group respectively. 